\title{
Factores de precariedad laboral y su relación con la salud de trabajadores en Bolivia
}

\author{
Ana María Arias-Urionaํ y Juan Carlos Ordóñez ${ }^{2}$
}

Forma de citar Arias-Uriona AM, Ordóñez JC. Factores de precariedad laboral y su relación con la salud de trabajadores asalariados y con contrato en Bolivia. Rev Panam Salud Publica. 2018;42:e98. https:/ /doi.org/10.26633/ RPSP.2018.98

RESUMEN Objetivo. Investigar la asociación de los factores de precariedad laboral con el uso de servicios de salud, consumo de tabaco y de alcohol entre trabajadores asalariados y con contrato en Bolivia.

Métodos. Estudio transversal con una muestra de trabajadores de entre 14 y 65 años, 1203 mujeres y de 1780 hombres, con datos de la Encuesta de Hogares 2015 de Bolivia. Las variables dependientes fueron el uso de servicios de salud, el consumo de tabaco y el consumo de alcohol. Las variables independientes fueron el salario, el tipo de contrato, las horas de trabajo y el ejercicio de derechos laborales. Se calcularon prevalencias y la asociación de los factores de precariedad laboral con las variables dependientes mediante modelos de regresiones binomiales multivariadas ajustadas por variables sociodemográficas. Los análisis fueron estratificados por sexo.

Resultados. Los modelos ajustados mostraron asociaciones significativas entre tener un contrato temporal y un menor uso de servicios de salud (USS) en hombres (razón de probabilidades [OR por sus siglas en inglés]: 0,70; intervalo de confianza de 95\% [IC95\%]: 0,56-0,87) y mujeres (OR: 0,62; IC95\%: 0,46-0,82). El bajo ejercicio de derechos estuvo también asociado a un menor USS (OR: 0,61; IC95\%: 0,45-0,83) en hombres. Las horas de trabajo prolongadas se asociaron al mayor consumo de alcohol en mujeres (OR: 1,75; IC95\%: 1,34-2,29). El salario bajo estuvo asociado al menor consumo de tabaco tanto en hombres (OR: 0,75; IC95\%: 0,57-0,99) como en mujeres (OR; 0,57; IC95\%: 0,33-0,99). Sin embargo, ser trabajador manual se asoció al consumo de tabaco (OR: 1,36; IC95\%: 1,09-1,70) entre los hombres.

Conclusiones. Tener un contrato temporal y pertenecer al quintil I (inferior) de ingresos se asocia a la menor utilización de servicios de salud y menor probabilidad de fumar en personas de uno y otro sexo. El horario prolongado de trabajo se asocia a mayor consumo de alcohol entre las mujeres. El bajo ejercicio de derechos estuvo asociado a un menor USS en hombres.

Palabras clave Empleo precario; determinantes sociales de la salud; uso de servicios de salud; consumo de tabaco; consumo de bebidas alcohólicas.

\footnotetext{
Escola Nacional de Saúde Pública, Lisboa, Portugal. Enviar correspondencia a Ana Maria Arias-Uriona: amfau79@gmail.com

2 Facultad de Tecnología, Universidad Mayor de San Andrés, La Paz, Bolivia.
}

Las condiciones de empleo y de trabajo son importantes determinantes sociales de la salud de los trabajadores y de sus familias $(1,2)$. Su estudio ha sido de gran interés para la salud pública durante la última década $(3,4)$ debido a la globalización del mercado, que genera diferentes formas de precariedad laboral

Este es un artículo de acceso abierto distribuido bajo los términos de la licencia Creative Commons Attribution-NonCommercial-NoDerivs 3.0 IGO, que permite su uso, distribución y reproducción en cualquier medio, siempre que el trabajo original se cite de la manera adecuada. No se permiten modificaciones a los artículos ni su uso comercial. Al reproducir un artículo no debe haber ningún indicio de que la OPS o el artículo avalan a una organización o un producto específico. El uso del logo de la OPS no está permitido. Esta leyenda debe conservarse, junto con la URL original del artículo. 
y nuevas formas de riesgo ocupacional, sobre todo en los países menos desarrollados $(5,6)$. A nivel micro, las condiciones de empleo están determinadas por los contratos de trabajo sujetos a leyes de trabajo (2). Sin embargo, en los países de mediana y baja renta, la mayoría de las condiciones de trabajo no están explícitamente reguladas por contratos $y$ existen altas proporciones de empleo informal (4). Por otra parte, a nivel macro, las condiciones de empleo están determinadas por los procesos económicos, sociales y políticos regulados por las políticas del mercado laboral y de protección social que, a su vez, determinan las desigualdades de salud $(2,7)$. La precarización afecta a todo tipo de trabajadores, también a los que cuentan con un trabajo estable (1).

Siguiendo la línea de Benach et al. (1), este estudio entiende que el empleo precario es un constructo multidimensional que incluye aspectos como la inseguridad laboral, el nivel de salario bajo, la privación económica y una protección social limitada, así como un bajo ejercicio de los derechos laborales. Estos factores, junto al estrés laboral y el horario prolongado de trabajo, guardan relación con la salud física y mental y con conductas de riesgo de los trabajadores $(4,8)$. Por ejemplo, la inseguridad laboral o el horario prolongado de trabajo se han asociado a un mayor riesgo de padecer enfermedades cardiovasculares $(9,10)$ y a estilos de vida no saludables como el tabaquismo (10-13) y el consumo de alcohol (11, 13-15).

Bolivia es un país que, en las últimas décadas, ha tenido una transformación social, política y económica con mejoras en el desarrollo humano y en la movilidad social en las áreas urbanas. Sin embargo, la estructura productiva débil e informal del país tiene como consecuencia una baja calidad laboral (16). Según datos oficiales del Censo 2012, solo 41,5\% de la población económicamente activa es asalariada y $18 \%$ es asalariada con contrato permanente. Las principales actividades económicas son la agricultura, ganadería, pesca, minería, industria manufacturera y el comercio al por mayor y menor (17). Los datos del año 2015 mostraban que alrededor de $30 \%$ de la población ocupada en regiones metropolitanas tenía contrato de trabajo, $60 \%$ tenía ingresos laborales por encima del salario mínimo de 240 dólares estadounidenses (USD por sus siglas en inglés), solo $28 \%$ contaba con seguro de salud, 19\% aportaba a las administradoras de fondos de pensiones (AFP) y 43\% trabajaba más de 48 horas (16).

El propósito de este estudio es investigar por primera vez la prevalencia de factores de precariedad laboral como el tipo de contrato, salario mensual, las horas de trabajo y el ejercicio de derechos, así como su asociación con la utilización de servicios de salud y el consumo de tabaco y de alcohol entre los trabajadores asalariados con contrato en Bolivia.

\section{MÉTODOS}

\section{Diseño, población de estudio, muestra y fuente de información}

Se realizó un estudio transversal en la población ocupada de 14 a 65 años de edad residente en Bolivia. Los datos se obtuvieron de la Encuesta de Hogares 2015, una encuesta transversal y representativa de la población residente en hogares de Bolvia, realizada anualmente por el Instituto Nacional de Estadística de Bolivia desde el año 1999 (18). Esta encuesta tiene como objetivo suministrar información sobre las condiciones de vida de los hogares, a partir de la recopilación de información de variables socioeconómicas y demográficas de la población boliviana (por ejemplo, características de la vivienda, salud, educación y condiciones laborales), las cuales permiten realizar un seguimiento a los Objetivos del Milenio establecidos por la Organización de las Naciones Unidas (ONU) (19). Se encuestaron 10404 viviendas ubicadas en 867 manzanas correspondientes a las unidades primarias de muestreo del área rural y urbana de los nueve departamentos de Bolivia $(18,20)$. La metodología aplicada para la recolección de la información fue la entrevista directa, conducida por personal capacitado que visitó las viviendas seleccionadas durante el período de recolección de información $(18,20)$.

Para el propósito de este estudio, se ha incluido a los trabajadores asalariados de 14 a 65 años de edad y con contrato laboral. La muestra final fue de 2983 individuos (1 203 mujeres y 1780 hombres).

\section{Variables}

En cuanto a las variables dependientes, la utilización de servicios de salud fue evaluado preguntando a los encuestados si habían acudido a algún servicio de salud por problemas de salud en el último año. La variable fue codificada en $0=\mathrm{No}$ y 1 = Sí (seguros de salud/establecimientos públicos o privados).

El consumo de tabaco fue evaluado a partir de la pregunta: "¿Durante los últimos 12 meses ha fumado cigarrillos?" Esta variable fue dicotomizada en $0=$ No/Ocasionalmente y 1 = Sí (Diariamente/Una o dos veces por semana/ Una o dos veces por mes).

El consumo de alcohol fue evaluado a partir de la pregunta: “¿Durante los últimos 12 meses ha consumido bebidas alcohólicas?" Esta variable fue dicotomizada en $0=$ No (Ninguna/Ocasionalmente) y 1 = Sí (Diariamente/Una o dos veces por semana/Una o dos veces por mes).

Las variables independientes principales fueron las relacionadas con las características y condiciones laborales.

El salario mensual se dividió en cinco quintiles y se creó una nueva variable "Quintil I de salario inferior" y se la dicotomizó en $0=$ Sí < 365 USD (quintil I) y $1=$ No $>365$ USD (quintiles II al V).

El tipo de contrato se obtuvo preguntando acerca del tipo de contrato firmado con el empleador, se dicotomizó la variable en $0=$ contrato temporal y $1=$ contrato permanente.

Las horas de trabajo se obtuvieron a partir de la variable "total de horas trabajadas a la semana" incluida en la base de datos. Se categorizó la variable en: $0=\leq 20$ horas, $1=21-48$ horas $\mathrm{y}$ $2>48$ horas. Luego, se creó una nueva variable "Horario prolongado de trabajo" y se la dicotomizó en: $0=$ No ( $\leq 40$ horas) y 1 = Sí ( $>40$ horas).

El ejercicio de derechos laborales se obtuvo a partir de la suma de las siguientes variables: recibió pago de aguinaldo, recibió pago de horas extras, tiene vacaciones y cuenta con un seguro de salud. Se creó una nueva variable, ejercicio de derechos 1 , y se la dicotomizó en 0 = Bajo (sin ejercicio o con ejercicio de dos derechos) y Alto = Sí (ejercicio de tres o cuatro de estos derechos).

Las variables de ajuste fueron la edad, el nivel de educación, la pertenencia a un grupo étnico indígena $u$ originario, el estado civil, el área de residencia y la presencia de enfermedades crónicas. La ocupación fue dicotomizada según la clasificación internacional de la Organización Internacional del Trabajo (CIUO-08) en $0=$ Trabajadores no manuales (Categorías OC1-5) y 1 = Trabajadores manuales (OC6-9) (21). 


\section{Análisis de los datos}

Todos los análisis fueron separados por sexo. Se realizó un análisis descriptivo de todas las variables y se calcularon las diferencias entre hombres y mujeres mediante la prueba de chi al cuadrado (cuadro 1). Se realizó un análisis de asociación bivariado entre cada una de las variables independientes y las dependientes mediante la prueba de chi cuadrado (cuadros 2 y 3). Para analizar la asociación multivariada se calcularon las razones de momios (OR por sus siglas en inglés) entre los individuos en condiciones precariedad y los que no lo están mediante dos modelos de regresión logística para cada variable dependiente. El primer modelo fue ajustado por la edad (variable continua) y el segundo modelo fue ajustado por la edad, el nivel de educación, la ocupación, el grupo étnico, el estado civil, el área de residencia y la presencia de enfermedades crónicas (cuadros 2 y 3). La validez de los modelos se evaluó mediante la prueba de bondad de HosmerLemeshow. Se comprobó que no hubiera problemas de multicolinealidad con el coeficiente de correlación de Pearson $(<0,6)$. Al existir correlación entre la clase social ocupacional y el nivel de educación, se escogió a la primera para el análisis.

\section{Consideraciones éticas}

El origen de los datos secundarios utilizados para este estudio es anónimo; los datos están disponibles en forma pública y gratuita en la página web del Instituto Nacional de Estadística (INE) de Bolivia. Es por ello que no se requiere aprobación de un comité de ética.

\section{RESULTADOS}

Los resultados del análisis descriptivo con las prevalencias de todas las variables para cada sexo se muestran en el cuadro 1. La media de edad fue de aproximadamente 38 años en ambos sexos. Se halló una mayor prevalencia de trabajadores manuales en los hombres $(50,1 \%)$ que en las mujeres $(23,9 \%)$, una mayor prevalencia de mujeres ubicadas en el quintil I inferior de salario menor a 365 USD $(31,4 \%)$ que de hombres $(20,7 \%)$. Entre los hombres se observaron prevalencias mayores de contratos temporales $(59,2 \%)$ y de ejercicio de
CUADRO 1. Características sociodemográficas de la población de estudio, presencia de enfermedad crónica y prevalencias de uso de servicios de salud, consumo de tabaco y consumo de alcohol, según el sexo

\begin{tabular}{|c|c|c|c|c|c|}
\hline \multirow{2}{*}{ Parámetros } & \multirow{2}{*}{$\begin{array}{c}\text { Mujeres } \\
(\mathrm{n}=1203 ; 40,3 \%) \\
\mathrm{n}\end{array}$} & \multicolumn{3}{|c|}{ Hombres ( $\mathrm{n}=1780 ; 59,7 \%)$} & \multirow[t]{2}{*}{ Valor de $P$} \\
\hline & & $\%$ & $n$ & $\%$ & \\
\hline \multicolumn{6}{|l|}{ Edad (años) } \\
\hline $14-23$ & 88 & 7,3 & 152 & 8,5 & 0,179 \\
\hline $24-33$ & 402 & 33,4 & 544 & 30,6 & \\
\hline $34-43$ & 366 & 30,4 & 520 & 29,2 & \\
\hline $44-53$ & 229 & 19,0 & 355 & 19,9 & \\
\hline$>54$ & 118 & 9,9 & 209 & 11,8 & \\
\hline Casos perdidos & 0 & 0,0 & 0 & 0,0 & \\
\hline Media & 37,58 & NA & 38,19 & NA & \\
\hline \multicolumn{6}{|l|}{ Nivel de educación } \\
\hline Primario incompleto & 13 & 1,1 & 6 & 0,3 & $\leq 0,001$ \\
\hline Primario & 64 & 5,3 & 116 & 6,5 & \\
\hline Secundario & 252 & 20,9 & 459 & 34,3 & \\
\hline Terciario & 874 & 72,7 & 1047 & 58,9 & \\
\hline Casos perdidos & 0 & 0,0 & 0 & 0,0 & \\
\hline \multicolumn{6}{|l|}{ Estado civil } \\
\hline Soltero/a & 558 & 46,4 & 511 & 28,7 & $\leq 0,001$ \\
\hline Casado/a o vive con la pareja & 645 & 53,6 & 1269 & 71,3 & \\
\hline Casos perdidos & 0 & 0,0 & 0 & 0,0 & \\
\hline \multicolumn{6}{|l|}{ Pertenencia a grupo étnico } \\
\hline Sí & 1002 & 83,4 & 1373 & 77,1 & $\leq 0,001$ \\
\hline No & 199 & 16,5 & 403 & 22,6 & \\
\hline Casos perdidos & 2 & 0,1 & 4 & 0,3 & \\
\hline \multicolumn{6}{|c|}{ Área geográfica de residencia } \\
\hline Rural & 1110 & 7,7 & 166 & 9,3 & 0,129 \\
\hline Urbana & 93 & 92,3 & 1614 & 90,7 & \\
\hline Casos perdidos & 0 & 0,0 & 0 & 0,0 & \\
\hline \multicolumn{6}{|l|}{ Enfermedad crónica } \\
\hline Sí & 136 & 11,3 & 133 & 7,5 & $\leq 0,001$ \\
\hline No & 1067 & 88,7 & 1647 & 92,5 & \\
\hline Casos perdidos & 0 & 0,0 & 0 & 0,0 & \\
\hline \multicolumn{6}{|l|}{ Ocupación } \\
\hline Trabajador/a manual & 287 & 23,9 & 872 & 50,1 & $\leq 0,001$ \\
\hline Trabajador/a no manual & 914 & 76,0 & 867 & 49,9 & \\
\hline Casos perdidos & 2 & 0,1 & 0 & 0,0 & \\
\hline \multicolumn{6}{|l|}{ Quintil I de salario ${ }^{a}$} \\
\hline Sí & 378 & 31,4 & 368 & 20,7 & $\leq 0,001$ \\
\hline No & 825 & 68,6 & 1412 & 79,3 & \\
\hline Casos perdidos & 0 & 0,0 & 0 & 0,0 & \\
\hline \multicolumn{6}{|l|}{ Tipo de contrato de trabajo } \\
\hline Temporal & 701 & 58,3 & 1053 & 59,2 & 0,629 \\
\hline Permanente & 502 & 41,7 & 727 & 40,8 & \\
\hline Casos perdidos & 0 & 0,0 & 0 & 0,0 & \\
\hline \multicolumn{6}{|l|}{ Horas de trabajo } \\
\hline$\leq 20$ horas & 109 & 9,1 & 53 & 3,0 & $\leq 0,001$ \\
\hline $21-40$ & 603 & 50,1 & 580 & 32,6 & \\
\hline$>40$ & 490 & 40,8 & 1147 & 64,4 & \\
\hline Media & 41,32 & NA & 48,78 & NA & \\
\hline Casos perdidos & 1 & 0,0 & 0 & 0,0 & \\
\hline \multicolumn{6}{|c|}{ Ejercicio de derechos laborales } \\
\hline Bajo & 206 & 17,1 & 243 & 13,7 & $\leq 0,01$ \\
\hline Alto & 997 & 82,9 & 1537 & 86,3 & \\
\hline Casos perdidos & 0 & 0,0 & 0 & 0,0 & \\
\hline
\end{tabular}

(Cont.) 
CUADRO 1. (Cont.)

\begin{tabular}{|c|c|c|c|c|c|}
\hline \multirow{2}{*}{ Parámetros } & \multirow{2}{*}{$\begin{array}{c}\text { Mujeres } \\
(n=1203 ; 40,3 \%) \\
n\end{array}$} & \multicolumn{3}{|c|}{ Hombres $(n=1780 ; 59,7 \%)$} & \multirow[t]{2}{*}{ Valor de $P$} \\
\hline & & $\%$ & $n$ & $\%$ & \\
\hline \multicolumn{6}{|c|}{ Uso de servicios de salud } \\
\hline Sí & 478 & 39,7 & 846 & 47,5 & $\leq 0,001$ \\
\hline No & 725 & 60,3 & 934 & 52,5 & \\
\hline Casos perdidos & 0 & 0,0 & 0 & 0,0 & \\
\hline \multicolumn{6}{|c|}{ Consumo de tabaco } \\
\hline Sí & 104 & 8,6 & 558 & 31,3 & $\leq 0,001$ \\
\hline No & 1099 & 91,4 & 1222 & 68,7 & \\
\hline Casos perdidos & 0 & 0,0 & 0 & 0,0 & \\
\hline \multicolumn{6}{|c|}{ Consumo de alcohol } \\
\hline Sí & 363 & 30,2 & 749 & 42,1 & $\leq 0,001$ \\
\hline No & 840 & 69,8 & 1031 & 57,9 & \\
\hline Casos perdidos & 0 & 0,0 & 0 & 0,0 & \\
\hline
\end{tabular}

a Salario < 365 dólares estadounidenses.

NA, no aplica.

Fuente: Cuadro elaborado por los autores con datos obtenidos de la Encuesta de Hogares, Bolivia, 2015.

derechos laborales $(86,3 \%)$ que entre las mujeres $(58,3 \%$, y $82,9 \%$, respectivamente). Hubo un promedio de 48,7 horas trabajadas a la semana en hombres y 41,3 horas en mujeres, con mayores prevalencias de hombres que trabajaban más de 40 horas semanales $(64,4 \%)$ que en las mujeres $(40,8 \%)$. También se observaron prevalencias más altas entre los hombres de uso de servicios de salud $(47,5 \%)$, de consumo de tabaco $(31,8 \%)$ y de consumo de alcohol $(42,1 \%)$ con respecto a las encontradas en mujeres $(39,7 \%, 8,6 \%$ y $30,2 \%$ respectivamente). Todas las diferencias entre hombres y mujeres fueron estadísticamente significativas con excepción de la edad, el área de residencia y el tipo de contrato.

Los modelos de regresión para cada variable dependiente ajustados por la edad (modelo I) y ajustados por la edad y las demás variables sociodemográficas (modelo II) se incluyeron en los cuadros 2 y 3 . En las mujeres, los modelos II mostraron asociaciones significativas entre tener contrato temporal y el uso de los servicios de salud (OR: 0,62; intervalo de confianza de 95\% [IC95\%], IC95\%: 0,46-0,82), entre tener alguna enfermedad crónica y el uso de servicios de salud (OR: 4,46; IC95\%: 2,6$7,44)$, entre encontrarse en el quintil inferior de salario menor a 365 USD (quintil I) y el menor consumo de tabaco (OR: 0,57; IC95\%: 0,33-0,99) y trabajar más de 40 horas a la semana estuvo asociado al consumo de alcohol (OR: 1,75; IC95\%: 1,34-2,29).
En los hombres, encontrarse en el quintil inferior de salario menor a 365 USD (quintil I) se asoció a un menor uso de los servicios de salud (OR: 0,74; IC95\%: $0,58-0,95)$ al ajustar por la edad (modelo I). Al ajustar por las demás variables (modelo II), tener un salario inferior a 365 USD se asoció al menor consumo de tabaco (OR: 0,75; IC95\%: 0,57-0,99); sin embargo, ser trabajador manual (OR: 1,36; IC95\%: 1,09-1,70), pertenecer a un grupo étnico (OR: 1,39; IC95\%: 1,07-1,81) y residir un área rural (OR: 1,62; IC95\%: 1,15-2,27) se asociaron asociados a una mayor probabilidad de consumo de tabaco. También en los modelos II, tener un contrato temporal se asoció a un menor uso de los servicios de salud (OR: 0,70; IC95\%: 0,56-0,87), tener alguna enfermedad crónica se asoció a un mayor USS (OR: 4,21; IC95\%: 2,66-6,68) El bajo ejercicio de derechos también se asoció al menor uso de servicios de salud (OR: 0,61; IC95\%: 0,45-0,83).

\section{DISCUSIÓN}

En este estudio se halló una mayor prevalencia de mujeres ubicadas en el quintil I inferior de salario para la muestra $(<365 \mathrm{USD})$, pese a que las mujeres tuvieron mayor educación terciaria y trabajo no manual. Esto coincide con los datos de la Comisión Económica para América Latina y el Caribe (CEPAL) que muestran que las mujeres ganan 25,6\% menos que los hombres, pese a estar más cualificadas, es decir que la inversión en educación y capacitación profesional de las mujeres no las acerca al nivel de ingresos de los hombres con la misma formación (22). La prevalencia de contratos permanentes fue de alrededor de $40 \%$ en ambos sexos. Sin embargo, se debe hacer notar que esta muestra de estudio incluyó a los trabajadores asalariados y con contrato escrito y solo representó a $19 \%$ de la muestra de trabajadores en la Encuesta de Hogares 2015. De estos, 8\% contaba con un contrato permanente, datos que coinciden con los de otro estudio en Bolivia (16).

La elevada prevalencia de hombres que trabajaban más de 40 horas a la semana con un promedio de 48,7 horas coincide con los hallazgos de un estudio reciente en trabajadores (excepto agricultores) con contrato escrito en Argentina, Chile, Colombia, Uruguay y América Central. Este estudio, realizado con base en encuestas nacionales de condiciones de trabajo, encontró que $40 \%$ de las mujeres y $58 \%$ de los hombres trabajaban más de 40 horas a la semana (6). Datos de la CEPAL señalan también un promedio muy superior al límite de las 40 horas semanales en la mayoría de los países de la Región (23). Otro estudio realizado en regiones metropolitanas de Bolivia encontró que $43 \%$ de los trabajadores tenían jornadas que hacían superar las 48 horas semanales de trabajo (16). Pese a que las leyes nacionales son la herramienta para regular las horas de trabajo (6), en Bolivia, como en la mayoría de los países de la Región, el límite de horas legal de trabajo es de 48 horas semanales (24) con un promedio de 42 horas semanales, en comparación con el promedio de 37 horas semanales en Europa (23).

La prevalencia de utilización de servicios de salud fue más alta en hombres que en las mujeres; esto podría deberse a que hubo un alto porcentaje de trabajadores manuales que, se sabe, presentan mayor prevalencia de riesgos laborales ergonómicos y psicológicos y de problemas de salud relacionados con la actividad $(6,25)$. La prevalencia de consumo de alcohol fue más alta en hombres que en mujeres. Este estudio encontró menor prevalencia de consumo de tabaco en las mujeres $(8,6 \%)$, que coincide con la prevalencia entre las mujeres de países de la Región (9\%) (26) y la mayor prevalencia de consumo de tabaco asociada a hombres de países de ingreso medio y alto de América Latina (27). 


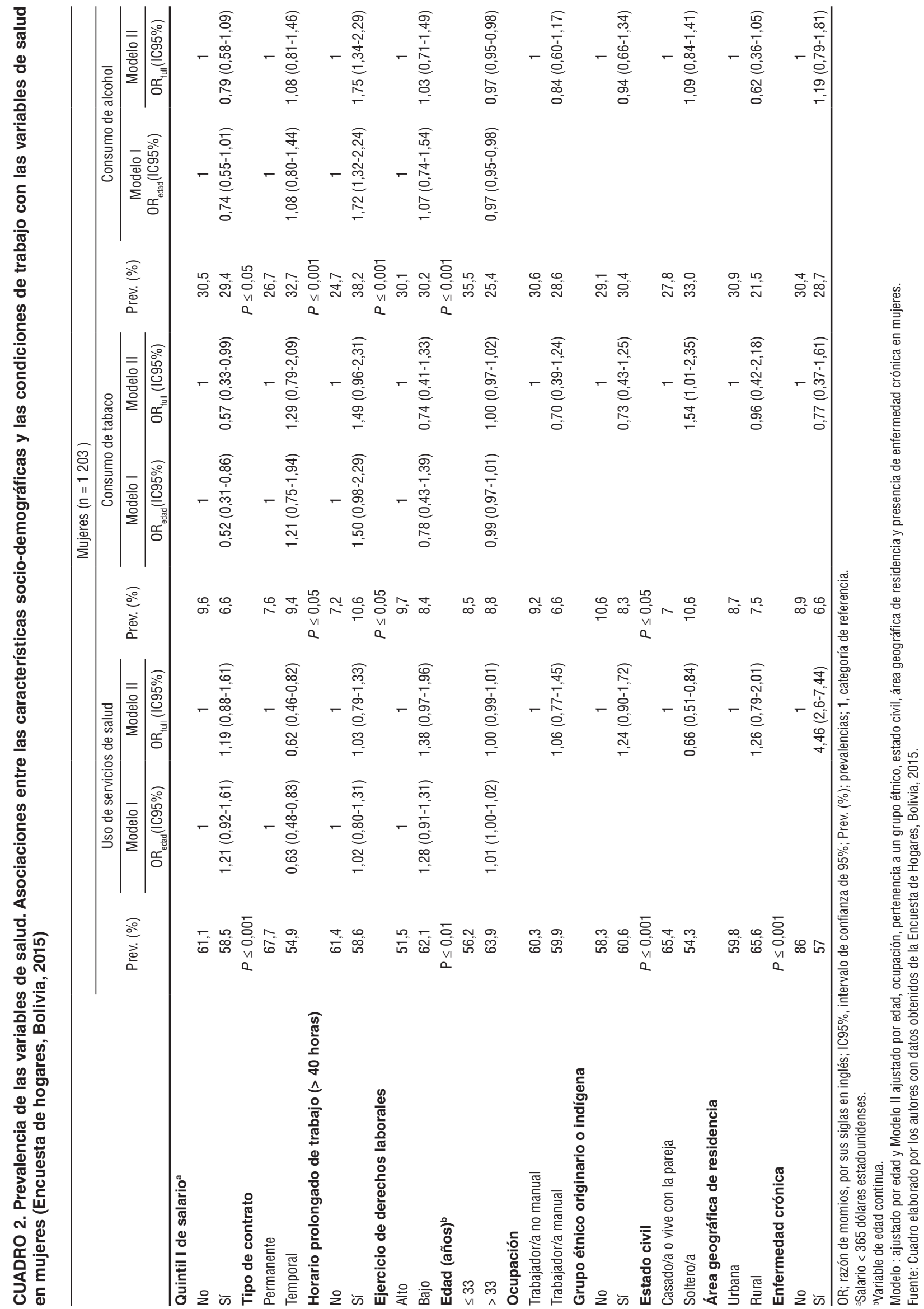




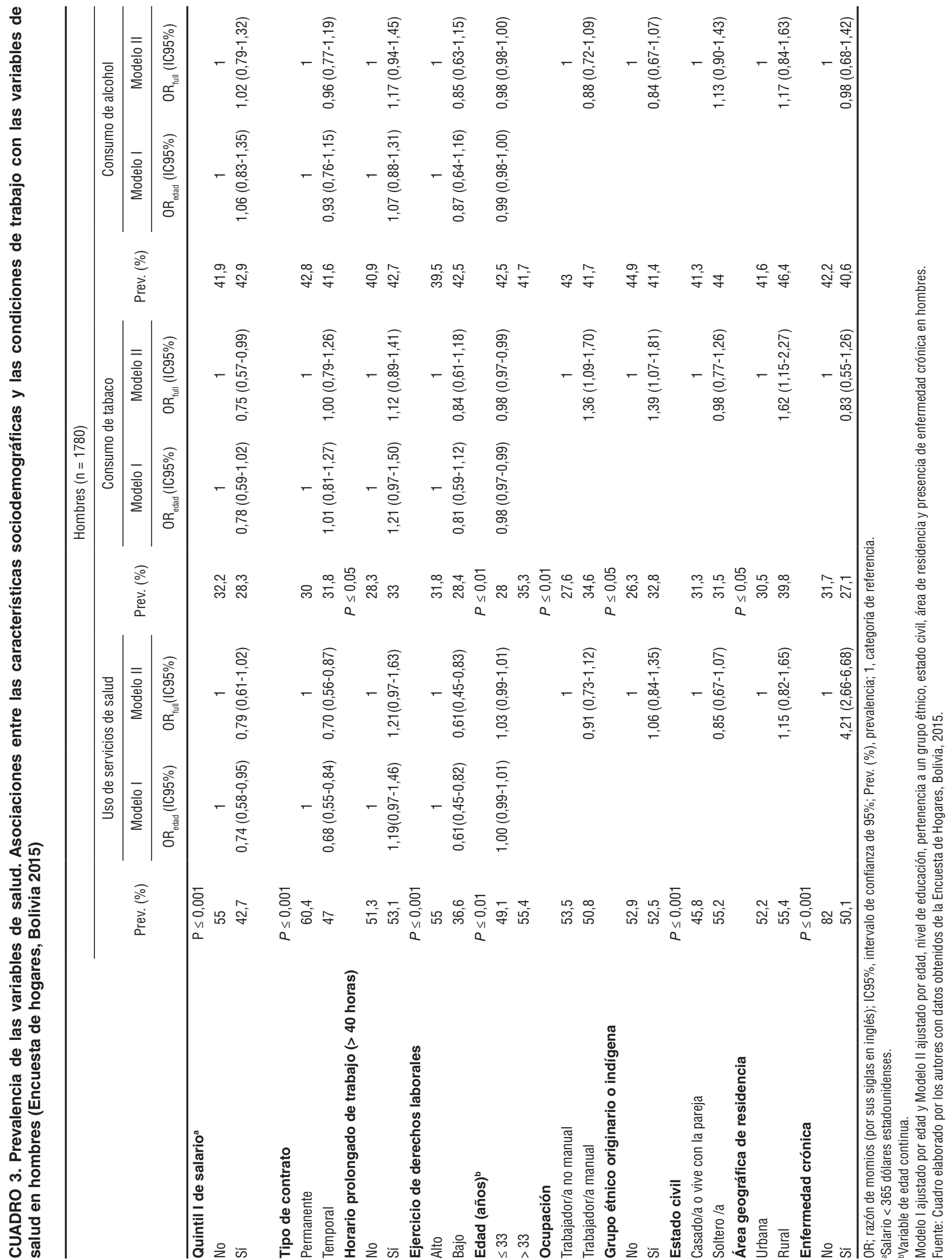


Los trabajadores con contrato temporal presentaron menor probabilidad de utilización de servicios de salud en ambos sexos. Este patrón desigual de utilización podría deberse a que existen empleados temporales que no cuentan con un seguro de salud pese a que, en Bolivia, los empleadores tienen la obligación de ofrecerle a sus trabajadores seguridad a corto plazo. Los trabajadores hombres con poco ejercicio de sus derechos laborales tuvieron también menor probabilidad de utilizar los servicios de salud; esta relación podría ser explicada porque estos trabajadores no contarían con un seguro de salud, tiempo (vacaciones) y menores recursos (al no recibir pago por horas extra y aguinaldos), factores que dificultarían el acceso a los servicios de salud.

Se ha encontrado que trabajar más de 40 horas a la semana estuvo relacionado a un mayor riesgo de consumo de alcohol entre las mujeres. Esto coincide con los hallazgos de una reciente revisión sistemática y metaanálisis que ha concluido que trabajadores con una jornada mayor a 48 horas tenían mayor riesgo de consumo de alcohol en Europa (13). Una explicación para esta relación sería que el alcohol alivia el estrés causado por las condiciones de trabajo; factores como la falta de control sobre el trabajo, la depresión, los desórdenes del sueño podrían contribuir también a esta relación $(13,28)$. Es importante mencionar que, aunque en nuestro estudio la prevalencia de horario prolongado fue menor entre las mujeres, se debe tomar en cuenta en este análisis la mayor carga de trabajo extra y no remunerado que ellas realizan en el hogar (23), indicador del que no se dispuso.

En nuestro estudio, los trabajadores de ambos sexos pertenecientes al quintil I de salario inferior (<365 USD) tuvieron menos probabilidades de consumir tabaco con respecto a los trabajadores que no pertenecían a este grupo y cuyo salario era superior (quintiles II al V). Esto coincide con algunos estudios en países en desarrollo, que encontraron que el consumo de tabaco se incrementaba de manera proporcional a los ingresos $(29,30)$. Sin embargo, este dato se debe interpretar con precaución, porque al observar las prevalencias de consumo de tabaco similares entre los trabajadores que pertenecían al grupo del quintil inferior y los que no pertenecían, se puede inferir que los individuos con menos ingresos (quintil I inferior) gastarían más dinero de su salario en tabaco en comparación con los individuos del grupo de ingresos más altos (quintiles II al V), aspecto que otros estudios han demostrado (29). Los trabajadores manuales hombres tuvieron mayor riesgo de consumo de tabaco; esto coincide con los resultados de otros estudios en países en desarrollo (26), en países de la Región (31) y en otros contextos (32). Las mayores prevalencias de estrés en los trabajadores manuales podría ser una explicación para esta relación, ya que este ha sido relacionado con un mayor riesgo de consumo de tabaco (33).

En nuestro conocimiento, este es el primer estudio en una muestra representativa de la población asalariada y con contrato escrito (sector formal) realizado en Bolivia que analiza el empleo precario tomando en cuenta diferentes dimensiones como el tipo de contrato, el ingreso, las horas de trabajo y el ejercicio de derechos laborales así como su relación con el uso de servicios de salud, tabaquismo y consumo de alcohol.

Este estudio tiene varias limitaciones. Al tratarse de un estudio de corte transversal, no es posible identificar causalidad entre nuestras variables de empleo precario y el uso de servicios de salud, el consumo de tabaco y de alcohol, o viceversa. Sin embargo, los resultados obtenidos en nuestro estudio fueron coincidentes con estudios previos. En este estudio solo se incluyeron trabajadores asalariados y con contrato de trabajo escrito, por lo que son necesarios futuros estudios que incluyan o que estén enfocados al estudio de los trabajadores informales, con trabajo no remunerado, o ambas condiciones, que no cuentan con protección social ni derechos laborales y entre los cuales se podrían encontrar las personas con peor salud que hayan sido expulsadas del mercado laboral. La encuesta utilizada para nuestro estudio no incluía indicadores del estado de salud físico, por lo que este estudio estuvo limitado en su análisis sobre la salud de los trabajadores. También hubo limitaciones en el análisis del consumo de alcohol y de tabaco, ya que solo se contaba con el indicador de frecuencia de consumo y no de cantidad de alcohol ingerido o del número de cigarrillos por día. Es importante tener en cuenta que, al tratarse de una encuesta, pudo existir un reporte inferior de consumo de alcohol y tabaco por parte de los encuestados. Por último, aunque nuestro análisis fue separado por sexo, esto no es suficiente para entender las inequidades de género.

\section{CONCLUSIONES}

Este estudio encontró que la precariedad laboral se asocia al uso de servicios de salud, al consumo de tabaco y de alcohol de los trabajadores. Al ajustar los análisis multivariados por las variables sociodemográficas, la mayor cantidad de horas de trabajo estuvieron relacionadas con la mayor frecuencia de consumo de alcohol en las mujeres, contar con un contrato temporal se asoció a una menor probabilidad de utilización de servicios de salud (USS) en ambos sexos. El bajo ejercicio de derechos laborales estuvo asociado a una menor probabilidad de USS en los hombres. Los trabajadores de ambos sexos ubicados en el quintil inferior de salario (<365 USD) tuvieron menor riesgo de consumo de tabaco. Sin embargo, entre los hombres, los trabajadores manuales, los que pertenecían a un grupo étnico indígena y los que residían en el área rural presentaron mayor probabilidad de consumo de tabaco. Se necesitan más estudios sobre la precariedad laboral para identificar los factores relacionados con la mala salud y con los hábitos de vida no saludables de los trabajadores. También son necesarios para realizar una vigilancia de la precariedad a través del tiempo, elaborar intervenciones para la prevención y cese del consumo de tabaco y alcohol y para influenciar sobre las políticas públicas en Bolivia, cuestiones importantes para poder mantener una fuerza laboral saludable.

Agradecimientos Los autores expresan su agradecimiento a Edgar Arias por su valiosa colaboración en la redacción de este artículo.

Conflicto de intereses Ninguno declarado por los autores.

Declaración Las opiniones expresadas en este manuscrito son responsabilidad del autor y no reflejan necesariamente los criterios ni la política de la RPSP/PAJPH y/o de la OPS. 


\section{REFERENCIAS}

1. Benach J, Vives A, Tarafa G, Delclos C, Muntaner C. What should we know about precarious employment and health in 2025? Framing the agenda for the next decade of research. Int J Epidemiol. 2016;45(1):232-8.

2. Benach J, Muntaner C. Employment and working conditions as health determinants. In: Commission of Social Determinants of Health, Lee JH, Sadana R, editors. Improving Equity in Health by Addressing Social Determinants: The World Health Organization; 2011:165-95.

3. Quinlan M, Mayhew C, Bohle P. The global expansion of precarious employment, work disorganization, and consequences for occupational health: a review of recent research. Int J Health Serv. 2001;31(2): 335-414.

4. Benach J, Vives A, Amable M, Vanroelen C, Tarafa G, Muntaner C. Precarious employment: understanding an emerging social determinant of health. Annu. Rev. Public Health. 2014;35:229-53.

5. Kawachi I. Globalization and workers' health. Ind Health 2008;46:421-3.

6. Merino-Salazar P, Artazcoz L, Cornelio C, Iniguez MJI, Rojas M, Martinez-Inigo D, et al. Work and health in Latin America: results from the working conditions surveys of Colombia, Argentina, Chile, Central America and Uruguay. Occup Environ Med. 2017;74(6):432-9.

7. Muntaner C, Chung H, Solar O, Santana V, Castedo A, Benach J. A macro-level model of employment relations and health inequalities. Int J Health Serv. 2010;40(2): 215-21.

8. CSDH. Closing the gap in a generation. Health equity through action on the social determinants of health: Final Report of the Commission on Social Determinants of Health. Geneva: World Health Organization; 2008, 232p.

9. Kivimaki M, Leino-Arjas P, Luukkonen R, Riihimaki H, Vahtera J, Kirjonen J. Work stress and risk of cardiovascular mortality: prospective cohort study of industrial employees. BMJ. 2002;325(7369):857.

10. Virtanen M, Nyberg ST, Batty GD, Jokela M, Heikkila K, Fransson EI, et al. Perceived job insecurity as a risk factor for incident coronary heart disease: systematic review and meta-analysis. BMJ. 2013;347:f4746.

11. Heikkila K, Fransson EI, Nyberg ST, Zins $\mathrm{M}$, Westerlund $\mathrm{H}$, Westerholm $\mathrm{P}$, et al. Job strain and health-related lifestyle: findings from an individual-participant meta-analysis of 118,000 working adults. Am J Public Health. 2013;103(11):2090-7.

12. Schulte PA, Pandalai S, Wulsin V, Chun H. Interaction of occupational and personal risk factors in workforce health and safety. Am J Public Health. 2012;102(3):434-48.

13. Virtanen M, Jokela M, Nyberg ST, Madsen IE, Lallukka T, Ahola K, et al. Long working hours and alcohol use: systematic review and meta-analysis of published studies and unpublished individual participant data. BMJ. 2015;350:7772.

14. Marchand A. Alcohol use and misuse: what are the contributions of occupation and work organization conditions? BMC Public Health. 2008;8(333).

15. Colell E, Sanchez-Niubo A, Benavides FG, Delclos GL, Domingo-Salvany A. Work-related stress factors associated with problem drinking: A study of the Spanish working population. Am J Ind Med. 2014;57(7):837-46.

16. Programa de Naciones Unidas para el Desarrollo (PNUD). Informe Nacional sobre Desarrollo Humano en Bolivia. El nuevo rostro de Bolivia: transformación y metropolización. La Paz: PNUD;2015. Disponible en: http:/ / www.bo.undp.org/ content/dam/bolivia/docs/undp_bo_ IDH2016.pdf

17. Instituto Nacional de Estadística de Bolivia (INE). Un pincelazo a las estadísticas con base a datos de censos. Datos relevantes del Censo de Población y Vivienda 2012 y de la Boleta Comunal del Primer Censo Agropecuario del Estado Plurinacional. 2014. La Paz: Instituto Nacional de Estadística; 2014. Disponible en: http:// cedla.org/blog/grupopoliticafiscal/5650\# sthash.zDHmhNHp.dpbs

18. Estado Plurinacional de Bolivia. Encuesta De Hogares 2015 [Internet]. ILO Microdata Repository. Disponible en: http:/ / www.ilo. org/surveydata/index.php/catalog/1443/ study-description

19. Instituto Nacional de Estadística de Bolivia (INE). El INE emprende la Encuesta de Hogares para medir el índice de pobreza 2014. INE, nota de prensa. Disponible en: http://www.ine.gob.bo/sitio_EH/pdf/ NP_2014_168.pdf

20. Instituto Nacional de Estadística de Bolivia (INE). Director de INE supervise operativos de campo de la Encuesta de Hogares 2015. INE, nota de prensa. Disponible en: http://www.ine.gob.bo/sitio_EH/pdf/ SEGUNDA\%20NOTA.pdf

21. International Standard Classification of Occupations 2008 (ISCO-08). Eurostat RAMON, Reference and Management of Nomenclatures. Disponible en: http:// ec.europa.eu/eurostat/ramon/nomenclatures/index.cfm?TargetUrl=LST_NOM_ DTL\&StrNom $=$ CL_ISCO08\&StrLanguage Code $=$ EN\&IntPcKey $=\&$ StrLayoutCode $=$ HIERARCHIC

22. Comisión Económica para América Latina y el Caribe (CEPAL). Pese a avances en el nivel educacional de las mujeres, persiste brecha salarial de género en la región. CEPAL; 2016. Disponible en: https:// www.cepal.org/es/comunicados/peseavances-nivel-educacional-mujeres-persiste-brecha-salarial-genero-la-region

23. Comisión Económica para América Latina y el Caribe (CEPAL). Panorama Social de América Latina. Santiago de Chile: CEPAL;
2013. Disponible en: https://repositorio. cepal.org/bitstream/handle/11362/ 35904/S2013868_es.pdf;jsessionid = 51EBDE99F9B9BCE9D65D58968372A847? sequence $=1$

24. International Labour Organization. Conditions of Work and Employment Programme. Disponible en: http:/ / www.ilo. org/dyn/travail/travmain.byCountry2

25. Rojas M, Gimeno D, Vargas-Prada S, Benavides FG. Musculoskeletal pain in Central American workers: results of the First Survey on Working Conditions and Health in Central America. Rev Panam Salud Publica. 2015;38(2):120-8.

26. Pampel F. National Income, Inequality and Global Patterns of Cigarette Use. Soc Forces. 2007;86(2):445-66.

27. Bardach A, Perdomo HA, Gandara RA, Ciapponi A. Income and smoking prevalence in Latin America: a systematic review and meta-analysis. Rev Panam Salud Publica. 2016;40(4):263-71.

28. Kivimaki M, Nyberg ST, Batty GD, Fransson EI, Heikkila K, Alfredsson L, et al. Job strain as a risk factor for coronary heart disease: a collaborative meta-analysis of individual participant data. Lancet. 2012;380(9852):1491-7.

29. Vázquez-Segobia LA. El consumo de tabaco en los hogares en México: resultados de la Encuesta de Ingresos y Gastos de los Hogares,1984-2000. Salud Publ Mex. 2002;44(1):76-81.

30. Kaplan GA, Lynch JW. Socioeconomic considerations in the primordial prevention of cardiovascular disease. Preventive Medicine 1999;29:S30-S5.

31. Rocha KB, Muntaner C, Gonzalez Rodriguez MJ, Baksai PB, Vallebuona C, Borrell C, et al. Social class, health inequalities, and health-related behaviors of working people in Chile. Rev Panam Salud Publica. 2013;33(5):340-8.

32. McFadden E, Luben R, Wareham N, Bingham S, Khaw KT. Occupational social class, educational level, smoking and body mass index, and cause-specific mortality in men and women: a prospective study in the European Prospective Investigation of Cancer and Nutrition in Norfolk (EPIC-Norfolk) cohort. Eur. J. Epidemiol. 2008;23(8):511-22.

33. Heikkila K, Nyberg ST, Fransson EI, Alfredsson L, De Bacquer D, Bjorner JB, et al. Job strain and tobacco smoking: an individual-participant data meta-analysis of 166,130 adults in 15 European studies. PloS One. 2012;7(7):e35463.

Manuscrito recibido el 7 de noviembre de 2017. Aceptado para su publicación, tras revisión, el 4 de abril de 2018 
ABSTRACT

Factors involved in job insecurity and their relationship with the health of salaried workers and contract workers in Bolivia

Objective. To examine the association between factors involved in job insecurity and: health service use, tobacco use, and alcohol use among salaried workers and contract workers in Bolivia.

Methods. A cross-sectional study was performed with a sample of workers composed of 1203 women and 1780 men between the ages of 14 and 65 years using data from Bolivia's 2015 Household Survey. The dependent variables were health service use, tobacco use, and alcohol use; the independent variables were wages, type of contract, work hours, and exercise of workers' rights. Prevalence rates were calculated and the association between factors involved in job insecurity and the dependent variables was assessed using multivariate binomial regression models with adjustment for sociodemographic variables. The analyses were stratified by sex.

Results. The adjusted models showed a significant association between working under a temporary contract and lower use of health services among men (odds ratio [OR]: $0.70 ; 95 \%$ confidence interval [95\% CI]: 0.56-0.87) and women (OR: $0.62 ; 95 \%$ CI: 0.46-0.82). Poor exercise of workers' rights was also associated with lower use of health services (OR: 0.61; IC 95\%: 0.45-0.83) among men. Long work hours showed an association with greater use of alcohol among women (OR: 1.75; 95\% CI: 1.34-2.29). Low wages were associated with lower tobacco use among men (OR: $0.75 ; 95 \%$ CI: $0.57-0.99)$ and women (OR; 0.57; 95\% CI: 0.33-0.99). However, being a manual worker was associated with tobacco use (OR: 1.36; 95\% CI: 1.09-1.70) among men.

Conclusions. Having a temporary contract and belonging to income quintile I (the poorest) are both associated with lower use of health services and lower probability of smoking in individuals of either sex. Long work hours are associated with greater alcohol use among women. Poor exercise of workers' rights is associated with lower use of health services among men.

Keywords Precarious employment; social determinants of health; health care utilization; tobacco use; alcohol drinking.

RESUMO

\section{Relação entre condições precárias de trabalho e a saúde dos trabalhadores assalariados e contratados na Bolívia}

Palavras-chave
Objetivo. Investigar a associação entre fatores relativos a condições precárias de trabalho e a utilização de serviços de saúde, tabagismo e uso de álcool em trabalhadores assalariados e contratados na Bolívia.

Métodos. Estudo transversal realizado em uma amostra de trabalhadores, 1.203 do sexo feminino e 1.780 do sexo masculino, com idade de 14 a 65 anos, a partir de dados obtidos da Pesquisa Nacional por Amostra de Domicílios de 2015 na Bolívia. As variáveis dependentes foram utilização dos serviços de saúde, tabagismo e uso de álcool. As variáveis independentes foram salário, tipo do contrato de trabalho, horas da jornada de trabalho e exercício dos direitos trabalhistas. Foram calculadas prevalências e a associação dos fatores relativos às condições de trabalho com as variáveis dependentes com o uso de modelos de regressão binomial multivariada ajustados segundo variáveis sociodemográficas. As análises foram estratificadas por sexo.

Resultados. Os modelos ajustados demonstraram uma associação significativa entre ter um contrato de trabalho temporário e menor utilização dos serviços de saúde entre os trabalhadores do sexo masculino (razão de chances [OR] 0,70; intervalo de confiança de 95\% [IC95\%] 0,56-0,87) e do sexo feminino (OR 0,62; IC95\% 0,46-0,82). O pouco exercício dos direitos também foi associado a menor utilização dos serviços de saúde (OR 0,61; IC95\% 0,45-0,83) no sexo masculino. Longas jornadas de trabalho foram associadas a um maior uso de álcool no sexo feminino (OR 1,75; IC95\% 1,34-2,29). O salário baixo foi associado a uma proporção menor de tabagismo tanto no sexo masculino (OR 0,75; IC95\% 0,57-0,99) como no sexo feminino (OR 0,57; IC95\% 0,33-0,99). Porém, ser trabalhador braçal foi associado ao tabagismo (OR 1,36; IC95\% 1,09-1,70) no sexo masculino. Conclusões. Ter contrato de trabalho temporário e pertencer ao quintil I (inferior) de renda estão associados a menor utilização dos serviços de saúde e menor probabilidade de ser tabagista em trabalhadores de ambos os sexos. Longas jornadas de trabalho estão associadas a um maior uso de álcool no sexo feminino. O pouco exercício dos direitos trabalhistas foi associado a menor utilização dos serviços de saúde no sexo masculino.

Emprego precário; determinantes sociais da saúde;uso de serviços de saúde; uso de tabaco; consumo de bebidas alcoólicas. 